\title{
Face Recognition Difficulties in Children: Hits and/or Correct Rejections?
}

\section{Ahmed M Megreya*}

Department of Psychological Sciences, College of Education, Qatar University, Qatar

Face recognition difficulties during childhood are well-documented. However, mixed results were obtained regarding the precise nature of these difficulties using different experimental tasks. Using recognition memory paradigm (learning a set of faces followed by an old/new recognition test using the previously studies faces mixed with an equal set of distractors [1], children under the age of ten were found to have lower rates of hits (the correct identification of previously-seen faces) and correct rejections (the rejection of previously-unseen faces) than older individuals [2]. In contrast, using the eyewitness identification methodology (seeing a target person through a video or live staged crime followed by an identification test using two target-present and target-absent lineups [3], children over five years of age were found to produce a hit rate that is comparable to adults [4] but older children still produce lower correct rejections [5].

This contrast supports the independence of hits and correct rejections that was frequently reported in several face processing studies [6-8]. In addition, it has been attributed to a key difference between face recognition and eyewitness identification tasks [4]. While the former typically require observers to encode and remember the faces of many individuals, the latter usually involve only a single target identity. The delayed onset of adult-like rates of correct identifications in face recognition experiments might therefore reflect the increased demands of these tasks. This points to an increase in memory load capacity with age rather than an improvement in face identification ability per se [4]. However, Megreya and Bindemann [9] reported lower hits in children under the age of 10 years using a widely used face identification task $[10,11]$ in which only one target had to be encoded at a time and memory loads were minimized. Therefore, the delayed onset of adult-like identification rates in face recognition experiments cannot be attributed to the memory load of these tasks. Further research is required to examine why hits reach maturation later in the face recognition paradigm than in the eyewitness identification methodology.

Importantly, recent evidence indicates that face recognition problems in children are perceptual, rather than memorial [9]. Therefore, the difficulty in encoding unfamiliar faces in the first place, independent of any memory-related problems, may contribute to the eyewitness identification problems in children. This is important as researchers have already attempted to improve the reliability of eyewitness identifications in children by implementing procedural changes such as elimination lineups [12] or the inclusion of a "not sure" response option [13]. Therefore, any attempts to improve the accuracy of eyewitness identification in children also need to focus on assessing their ability to encode unfamiliar faces in the first place.

\section{References}

1. Megreya AM (2012) Accuracy of face recognition. In Adamo Quaglia and Calogera M Epifano (Eds). Face Recognition: Methods Applications and Technology New York Nova Publishers.

2. Shapiro PN, Penrod S (1986) Meta-analysis of facial identification studies Psychological Bulletin 100: 139-156.

3. Megreya AM, Memon A, Havard C (2012) The headscarf effect: Direct evidence from eyewitness identification paradigm. Applied Cognitive Psychology 26: 308-315.

4. Pozzulo JD, Lindsay R (1998) Identification accuracy of children versus adults: A meta-analysis. Law and Human Behavior 22: 549-570.

5. Havard C (2014) Are children less reliable at making visual identifications than adults? A review. Psychology Crime and Law 20: 372-388.

6. Megreya AM, Burton AM (2006b) Unfamiliar faces are not faces: Evidence from a matching task. Memory and Cognition 4: 865-876.

7. Megreya AM, Burton AM (2007) Hits and false positives in face matching: A familiarity based dissociation. Perception and Psychophysics 69: 1175-1184.

8. Megreya AM, Burton AM (2008) Matching faces to photographs: poor performance in eyewitness memory (without the memory). Journal of Experimental Psychology: Applied 14: 364-372.

9. Megreya AM, Bindemann M (2015) Developmental improvement and agerelated decline in unfamiliar face matching. Perception 44: 2-44.

10. Megreya AM, Bindemann M (2013) Individual differences in personality and face identification. Journal of Cognitive Psychology 25: 30-37.

11. Megreya AM, Sandford A, Burton AM (2013) Matching face images taken on the same day or months apart: The limitations of photo-ID. Applied Cognitive Psychology 27: 700-706.

12. Pozzulo JD, Lindsay $R$ (1999) Elimination lineups: An improved identification procedure for child eyewitnesses. Journal of Applied Psychology 84: 167-176.

13. Brewer N, Keast AL, SauerJ (2010) Children's eyewitness identification performance: Effects of a not sure response option and accuracy motivation. Legal and Criminological Psychology 15: 261-277.
*Corresponding author: Ahmed M Megreya, Department of Psychological Sciences, College of Education, Qatar University, Qatar, E-mail: amegreya@qu.edu.qa

Received September 23, 2015; Accepted September 24, 2015; Published September 29, 2015

Citation: Megreya AM (2015) Face Recognition Difficulties in Children: Hits and/or Correct Rejections? J Psychol Abnorm Child 4: e105. doi:10.4172/23299525.1000e105

Copyright: () 2015 Megreya AM. This is an open-access article distributed under the terms of the Creative Commons Attribution License, which permits unrestricted use, distribution, and reproduction in any medium, provided the original author and source are credited. 